\title{
Self-trapped electron states in nanotubes
}

\author{
L. Bratek, \\ Department of Mathematical Sciences, University of Durham, \\ Durham DH1 3LE, UK \\ L. Brizhik $†$ A. Eremko \\ Bogolyubov Institute for Theoretical Physics, 03143 Kyiv, Ukraine \\ B. Piette§, M. Watson, and W. Zakrzewski" \\ Department of Mathematical Sciences,University of Durham, \\ Durham DH1 3LE, UK
}

\begin{abstract}
We study numerically self-trapped (polaron) states of quasiparticles (electrons, holes or excitons) in a deformable nanotube formed by a hexagonal lattice, wrapped into a cylinder (carbon- and boron nitride-type nanotube structures). We present a Hamiltonian for such a system taking into account an electron-phonon interaction, and determine conditions under which the lowest energy states are polarons. We compute a large class of numerical solutions of this model for a wide range of the parameters. We show that at not too strong electron-phonon coupling, the system admits ring-like localized solutions wrapped around the nanotube (the charge carrier is localized along the nanotube axis and uniformly distributed with respect to the azimuthal coordinate). At stronger coupling, solutions are localized on very few lattice sites in both directions of the nanotube. The transition from one type solution to the other one depends on the diameter of the nanotube. We show that for the values of the carbon nanotube parameters, the polarons have a ring-like structure wrapped around the nanotube with a profile resembling that of the nonlinear Schrödinger soliton.
\end{abstract}

\section{Introduction}

Low-dimensional systems have been studied intensively during the last decades. Some systems have been found to possess unusual properties which turn out to be important for their practical applications in microelectronics and nanotechnologies. Conducting polymers, quasi-onedimensional organic and inorganic compounds are good examples of such systems [1]. Recently, nanotubes have become the subject of many intensive experimental and theoretical studies. In particular, carbon $[2,3,4]$ and boron nitride [5] nanotubes have been shown to be useful for miniaturised electronic, mechanical, electromechanical and optoelectronic devices. Some of the intriguing physical properties of such systems are due to the electron-phonon interactions which are particularly important in low-dimensional systems.

Although low-dimensional systems have been studied mainly within simplified single-chain (single-band) models resulting in the theoretical predictions of many striking phenomena $[6,7,8]$, many of them, such as Peierls transitions, formation of charge density waves, soliton states, etc have been verified experimentally in real substances. However, taking into account the more realistic complex structure of these substances is quite important for the proper description of

*e-mail address: Lukasz.Bratek@ifj.edu.pl

${ }^{\dagger}$ e-mail address: brizhik@bitp.kiev.ua

${ }^{\ddagger}$ e-mail address: eremko@bitp.kiev.ua

$\S$ e-mail address: B.M.A.G.Piette@durham.ac.uk

ฯe-mail address: m.j.watson@durham.ac.uk

"e-mail address: W.J.Zakrzewski@durham.ac.uk 
their properties and can result in qualitatively new features. This is particularly true in the case of nanotubes who possess series of energy bands which are determined by one-dimensional (1D) energy dispersion relations on the wave vector $k$ along the axis of the nanotube. It is well known that sufficiently long single wall carbon nanotubes (SWNT), in which half of the energy band states are occupied, possess properties of 1D-metals or semiconductors depending on their diameter and chirality $[2,3,9,10]$. In fact, their properties can be modified in a controllable way by varying either the nanotube diameter and chirality or by doping nanotubes with some impurity atoms, molecules or compounds [11].

In 1D systems the electron-phonon interactions can lead to the formation of stable selftrapped states with spontaneous symmetry-breaking distortions [8, 12]. Such soliton states are realized in quasi-1D organic and inorganic materials and can successfully describe various properties of conjugated polymers in the framework of the Su-Schrieffer-Heeger model [12]. The possibility for the formation of states with a spontaneously broken symmetry in carbon nanotubes has been discussed in $[10,13,14]$. In particular, large polarons (or solitons) in nanotubes have recently been considered in $[15,16]$. In these models the long-wave approximation has been used for the states close to the Fermi-level, which is equivalent to the continuum approximation. In this way, however, the essential details of the crystallographic structure of the system are not taken into account.

Here we study numerically the formation and properties of polaron states in zigzag nanotubes using the semi-empirical tight-binding model in which we consider only the nearest-neighbour hopping interactions [2]. The merit of this method is its simplicity, in spite of which it often gives a quantitative agreement with the experimental results. This has been demonstrated in [12] for conjugated polymers and for carbon nanotubes in [2, 3, 9, 10]. Moreover, we consider only the ground state of a single electron in a hexagonal nanotube. Such systems are already quite complicated and though such states in 1D chains have been studied intensively both analytically and numerically, little is known about them in the case of nanotubes.

\section{The Model}

We consider a model in which a two-dimensional sheet of hexagonally bound equivalent atoms (graphene-like sheet) is rolled-up into a zigzag nanotube. Calling $d$ the length of each side of the hexagons, $R$ the radius of the nanotube, $n$ the number of hexagonal cells wrapped around the nanotube, we define (cfr. Fig 1)

$$
\alpha=2 \pi / n, \quad a=d \sqrt{3}, \quad b=d / 2 .
$$

The site labelling that we use is shown in Fig.1 and coincides with the conventional one [2]. Here $j$ is an axial index, $i=1, \ldots, n$ is the index labelling the sites around the nanotube, and $\rho=1,2$ labels the two types of atoms (usually called A and B): those who have a nearest neighbour one site down $(\rho=0)$ and those who have a nearest neighbour one site 'up' $(\rho=1)$ - see Fig.1. Then the position of any nanotube lattice site, at its equilibrium, can be described by:

$$
\vec{R}_{i, j, \rho}^{0}=R \sin \left(\left(i+\frac{j+\rho}{2}\right) \alpha\right) \vec{e}_{x}+R \cos \left(\left(i+\frac{j+\rho}{2}\right) \alpha\right) \vec{e}_{y}+\frac{3 j+\rho}{2} d \vec{e}_{z} .
$$

In Fig. 2 we present a drawing of the horizontal cross-section of the nanotube. Note that the parameters $R, a$ and $\alpha$ are connected by

$$
a=4 R \sin \left(\frac{\alpha}{4}\right)
$$

Next we define

$$
\vec{U}_{i, j, \rho}=\vec{u}_{i, j, \rho}+\vec{s}_{i, j, \rho}+\vec{v}_{i, j, \rho}
$$




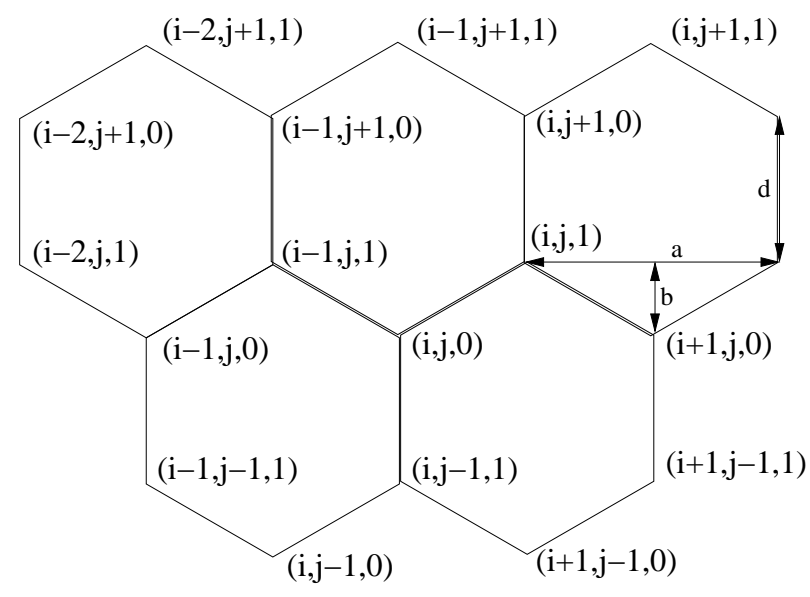

Figure 1: Hexagonal lattice, $j$ is assumed even.

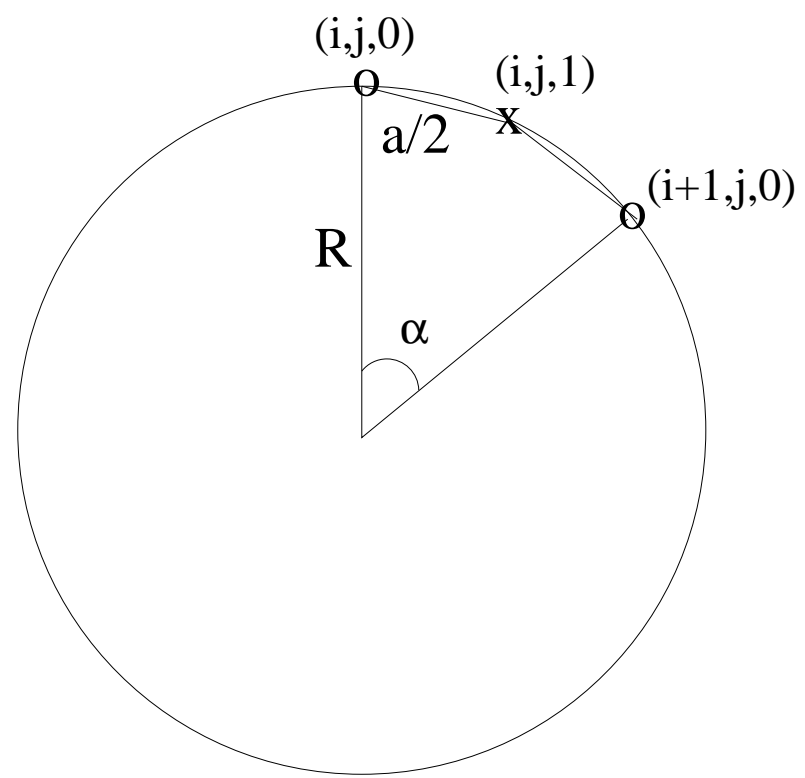

Figure 2: Cross section of the nanotube. 
as the displacement vector of the atoms from their equilibrium positions where $\vec{u}_{i, j, \rho}$ is tangent to the surface of the undeformed nanotube and perpendicular to the nanotube axis, $\vec{v}_{i, j, \rho}$ is tangent to this surface and parallel to the nanotube axis, and $\vec{s}_{i, j, \rho}$ is normal to the surface of the nanotube. Then, using Cartesian coordinates, we have

$$
\begin{aligned}
& \vec{u}_{i, j, \rho}=u_{i, j, \rho}\left(\cos ((i+(j+\rho) / 2) \alpha) \overrightarrow{e_{x}}-\sin ((i+(j+\rho) / 2) \alpha) \overrightarrow{e_{y}}\right), \\
& \vec{s}_{i, j, \rho}=s_{i, j, \rho}\left(\sin ((i+(j+\rho) / 2) \alpha) \overrightarrow{e_{x}}+\cos ((i+(j+\rho) / 2) \alpha) \overrightarrow{e_{y}}\right), \\
& \vec{v}_{i, j, \rho}=v_{i, j, \rho} \overrightarrow{e_{z}},
\end{aligned}
$$

where $u_{i, j, \rho}, s_{i, j, \rho}$ and $v_{i, j, \rho}$ are the corresponding amplitudes of displacements. So the positions of the lattice sites are given by

$$
\vec{R}_{i, j, \rho}=\vec{R}_{i, j, \rho}^{0}+\vec{U}_{i, j, \rho} .
$$

We also define the following lattice vectors connecting the atom $(i, j, \rho)$ with its three nearest neighbours $\delta(i, j, \rho)$ with $\delta=r, l, d$ for right $(r)$, left $(l)$ and down or up $(d)$ neighbours:

$$
\vec{D} \delta_{i, j, \rho}=\vec{R}_{\delta(i, j, \rho)}-\vec{R}_{i, j, \rho}
$$

When $\vec{U}_{i, j, \rho}=0$ we add the upper index 0 to all quantities to indicate their values at the equilibrium position. Hence $\vec{D} \delta_{i, j, \rho}=\overrightarrow{D \delta}_{i, j, \rho}^{0}+\overrightarrow{d \delta_{i, j, \rho}}$ with $\vec{d}_{i, j, \rho}=\vec{U}_{\delta(i, j, \rho)}-\vec{U}_{i, j, \rho}$. Note that

$$
\left|\overrightarrow{D r}_{i, j, \rho}^{0}\right|=\left|\overrightarrow{D l_{i, j, \rho}^{0}}\right|^{0}\left|\overrightarrow{D d_{i, j, \rho}^{0}}\right|=d .
$$

The potential energy of the lattice distortion includes central forces, which depend only on the distance between two sites. In the case of small displacement, i.e when $\left|\overrightarrow{d \delta}_{i, j, \rho}\right| \ll d$, the distance between lattice sites is approximately given by:

$$
\left|\vec{D} \delta_{i, j, \rho}\right| \approx d+W \delta_{i, j, \rho}
$$

where

$$
W \delta_{i, j, \rho}=\frac{\overrightarrow{d \delta_{i, j, \rho}} \cdot \overrightarrow{D \delta_{i, j, \rho}^{0}}}{d}
$$

are the changes of distances between nearest neighbours due to the displacements of the sites.

As is well known, the central forces between neighbouring sites do not provide lattice stability. Therefore, we introduce additional terms. First, assuming that the force between two atoms is not purely central, in addition to $W \delta_{i, j, \rho}$ which are invariant under translations, we introduce also the quantities $\Omega \delta_{i, j, \rho}$ which describe relative shifts of neighbouring atoms in a graphene sheet and which will be needed to remove the non-physical zero modes of the phonon Hamiltonian. The explicit expressions for these quantities are:

$$
\begin{aligned}
& \Omega r_{i, j, 0}=\frac{1}{2}\left(\cos \left(\frac{\alpha}{4}\right)\left(u_{i, j, 1}-u_{i, j, 0}\right)+\sin \left(\frac{\alpha}{4}\right)\left(s_{i, j, 1}+s_{i, j, 0}\right)\right)-\frac{\sqrt{3}}{2}\left(v_{i, j, 1}-v_{i, j, 0}\right), \\
& \Omega l_{i, j, 0}=\frac{1}{2}\left(\cos \left(\frac{\alpha}{4}\right)\left(u_{i, j, 0}-u_{i-1, j, 1}\right)+\sin \left(\frac{\alpha}{4}\right)\left(s_{i-1, j, 1}+s_{i, j, 0}\right)\right)-\frac{\sqrt{3}}{2}\left(v_{i-1, j, 1}-v_{i, j, 0}\right), \\
& \Omega d_{i, j, 0}=-u_{i, j-1,1}+u_{i, j, 0} \\
& \Omega r_{i, j, 1}=\Omega r_{i, j, 0}, \quad \Omega l_{i, j, 1}=\Omega l_{i+1, j, 0}, \quad \Omega d_{i, j, 1}=\Omega d_{i, j+1,0} .
\end{aligned}
$$

Next, as in $[17,18]$, we introduce also the force of bond-bending between the atoms induced by the deviation of the valence angles from their equilibrium values. For this we compute the solid angle spanned by the 3 lattice vectors located at a given site. Clearly, this angle is given by

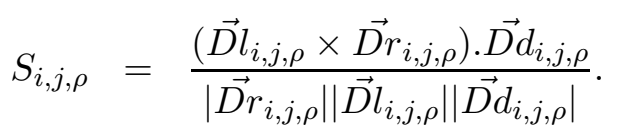


For small displacements we have

$$
S_{i, j, \rho} \approx S_{i, j, \rho}^{0}+\frac{\sqrt{3}}{2 d} C_{i, j, \rho}
$$

where $S_{i, j, \rho}^{0}=\frac{3}{4} \sin \left(\frac{\alpha}{2}\right)$ and

$$
\begin{aligned}
C_{i, j, 0}= & \frac{\sqrt{3}}{4} \sin \left(\frac{\alpha}{2}\right)\left(2 v_{i, j, 0}-v_{i, j, 1}-v_{i-1, j, 1}\right) \\
& -\cos \left(\frac{\alpha}{4}\right) s_{i, j-1,1}+3 \cos ^{3}\left(\frac{\alpha}{4}\right) s_{i, j, 0} \\
& +\left(\frac{3}{2} \cos \left(\frac{\alpha}{4}\right)-\frac{5}{2} \cos ^{3}\left(\frac{\alpha}{4}\right)\right)\left(s_{i-1, j, 1}+s_{i, j, 1}\right) \\
& +\sin \left(\frac{\alpha}{4}\right)\left(\frac{5}{2} \cos ^{2}\left(\frac{\alpha}{4}\right)-1\right)\left(u_{i, j, 1}-u_{i-1, j, 1}\right) \\
C_{i, j, 1}= & \frac{\sqrt{3}}{4} \sin \left(\frac{\alpha}{2}\right)\left(v_{i, j, 0}+v_{i+1, j, 0}-2 v_{i, j, 1}\right) \\
& -\cos \left(\frac{\alpha}{4}\right) s_{i, j+1,0}+3 \cos ^{3}\left(\frac{\alpha}{4}\right) s_{i, j, 1} \\
& +\left(\frac{3}{2} \cos \left(\frac{\alpha}{4}\right)-\frac{5}{2} \cos ^{3}\left(\frac{\alpha}{4}\right)\right)\left(s_{i+1, j, 0}+s_{i, j, 0}\right) \\
& +\sin \left(\frac{\alpha}{4}\right)\left(\frac{5}{2} \cos ^{2}\left(\frac{\alpha}{4}\right)-1\right)\left(u_{i+1, j, 0}-u_{i, j, 0}\right) .
\end{aligned}
$$

Taking into account the harmonic potential terms responsible for the central i.e $V_{W}$, noncentral, $V_{\Omega}$, and the bond-bending, $V_{C}$ forces, and using the approximation of the nearestneighbour interaction, we define the phonon Hamiltonian as:

$$
\begin{aligned}
H_{p h}= & \frac{1}{2} \sum_{i, j, \rho}\left(\frac{p_{i, j, \rho}^{2}}{M}+\frac{q_{i, j, \rho}^{2}}{M}+\frac{r_{i, j, \rho}^{2}}{M}+k_{c} C_{i, j, \rho}^{2}\right. \\
& \left.+k\left[W r_{i, j, \rho}^{2}+W l_{i, j, \rho}^{2}+W d_{i, j, \rho}^{2}+\Omega r_{i, j, \rho}^{2}+\Omega l_{i, j, \rho}^{2}+\Omega d_{i, j, \rho}^{2}\right]\right) .
\end{aligned}
$$

Here $p_{i, j, \rho}, q_{i, j, \rho}$ and $r_{i, j, \rho}$ are the momenta, canonically conjugate, respectively, to the displacements $u_{i, j, \rho}, s_{i, j, \rho}$ and $v_{i, j, \rho} . M$ is the atom mass, $k$ is the elasticity constant that characterises the central force, and $k_{c}$ is a characteristic constant of the bond-bending force.

Next we assume that each atom in the hexagonal lattice has a well isolated non-degenerate electron level $\mathcal{E}_{0}$ ( $\pi$-orbital in the case of a carbon nanotube) and we take into account the hopping interaction, $J$, of each atom only with its three nearest neighbours.

The electron-phonon interaction has different sources [10, 19, 13, 17, 20]. Often, the phonon modulation of the hopping interaction is invoked which, in the linear approximation with respect to the displacements, gives

$$
J_{(i, j, \rho) ; \delta(i, j, \rho)}=J-G_{2} W \delta_{i, j, \rho}
$$

thus leading to the electron phonon interaction proportional to $G_{2}$.

In general, as has been shown in [17], in addition to the conventional modulation of the hopping interaction one should also take into account the interaction between electrons and ions through the Coulomb potential which is modulated by the lattice vibrations.

The fields of neighbouring atoms then alter the local electron energy, $\mathcal{E}_{i, j, \rho}$, and so, in the same linear approximation, we can write

$$
\mathcal{E}_{i, j, \rho}=\mathcal{E}_{0}+\chi_{1}\left(W r_{i, j, \rho}+W l_{i, j, \rho}+W d_{i, j, \rho}\right)+\chi_{2} C_{i, j, \rho}
$$

where $\chi_{1}$ characterises the influence of the distance and $\chi_{2}$ the influence of the bond-bending deviations. 
The constants $G_{2}, \chi_{1}$ and $\chi_{2}$ determine the strength of the electron-phonon coupling. Their calculation would be the subject of microscopic considerations. The estimate of $G_{2}$ for carbon nanotubes was obtained in [21]. The coupling, through the Coulomb potential, provides an input into all these constants. Moreover, the curvature of the lattice is an important factor: the curvature-induced charge polarisation, present in carbon nanotubes, results in the vibration modulated dipole component of the Coulomb potential. As it was indicated in [22], where this fact had been taken into account through the Lydann-Sachs-Teller relation, such an electronphonon interaction is stronger that the one due to the deformational potential. In this paper we treat the constants $G_{2}, \chi_{1}$ and $\chi_{2}$ as parameters and we investigate the ground self-trapped state of an electron depending on the value of these parameters. The strength of the electron-phonon interaction plays a key role in many effects. When the coupling constant of this interaction is strong enough it can lead to the self-trapping of a quasiparticle. By 'quasiparticle' we denote an electron, a hole or an exciton in a nanotube.

The self-trapped states of quasiparticles are usually described in the adiabatic approximation which is equivalent to the semiclassical consideration in which the vibrational subsystem is described as a classical one. Therefore, the state of a quasiparticle in such a model is described by the Hamiltonian functional

$$
\begin{aligned}
\mathcal{H}= & H_{p h}+\sum_{i, j, \rho}\left(\mathcal{E}_{0}\left|\varphi_{i, j, \rho}\right|^{2}-\frac{1}{2} J\left(\varphi_{i, j, \rho}^{*} \varphi_{r(i, j, \rho)}+\varphi_{r(i, j, \rho)}^{*} \varphi_{i, j, \rho}\right.\right. \\
+ & \left.\varphi_{i, j, \rho}^{*} \varphi_{l(i, j, \rho)}+\varphi_{l(i, j, \rho)}^{*} \varphi_{i, j, \rho}+\varphi_{i, j, \rho}^{*} \varphi_{d(i, j, \rho)}+\varphi_{d(i, j, \rho)}^{*} \varphi_{i, j, \rho}\right) \\
+ & \chi_{1}\left|\varphi_{i, j, \rho}\right|^{2}\left(W r_{i, j, \rho}+W l_{i, j, \rho}+W d_{i, j, \rho}\right)+\chi_{2}\left|\varphi_{i, j, \rho}\right|^{2} C_{i, j, \sigma} \\
+ & \frac{1}{2} G_{2}\left[\left(\varphi_{i, j, \rho}^{*} \varphi_{r(i, j, \rho)}+\varphi_{r(i, j, \rho)}^{*} \varphi_{i, j, \rho}\right) W r_{i, j, \rho}+\left(\varphi_{i, j, \rho}^{*} \varphi_{l(i, j, \rho)}+\varphi_{l(i, j, \rho)}^{*} \varphi_{i, j, \rho}\right) W l_{i, j, \rho}\right. \\
& \left.\left.+\left(\varphi_{i, j, \rho}^{*} \varphi_{d(i, j, \rho)}+\varphi_{d(i, j, \rho)}^{*} \varphi_{i, j, \rho}\right) W d_{i, j, \rho}\right]\right) .
\end{aligned}
$$

Here $H_{p h}$ is given by (15) and $\varphi_{i, j, \rho}$ is the amplitude of the probability of quasiparticle being located on the site $(i, j, \rho)$.

From (18), after expanding the $W$ 's, $\Omega$ 's and $C$ 's, we can derive the following static equations for the functions $\varphi_{i, j, \rho}, u, v$ and $s$ :

$$
\begin{gathered}
0=\left(\mathcal{W}+\mathcal{E}_{0}\right) \varphi-J\left(\varphi_{r}+\varphi_{l}+\varphi_{d}\right)+\chi_{1} \varphi\left(W_{r}+W_{l}+W_{d}\right)+\chi_{2} \varphi C \\
+G_{2}\left[\varphi_{r} W_{r}+\varphi_{l} W_{l}+\varphi_{d} W_{d}\right] \\
0=k\left[\sqrt{3} \cos \left(\frac{\alpha}{4}\right)\left(W_{l}-W_{r}\right)+\cos \left(\frac{\alpha}{4}\right)\left(\Omega_{l}-\Omega_{r}\right)+2 \Omega_{d}\right]+k_{c}\left[\sin \left(\frac{\alpha}{4}\right)\left(\frac{5}{2} \cos ^{2}\left(\frac{\alpha}{4}\right)-1\right)\left(C_{l}-C_{r}\right)\right] \\
+\chi_{1}\left(\left|\varphi_{l}\right|^{2}-\left|\varphi_{r}\right|^{2}\right)\left(\frac{\sqrt{3}}{2} \cos \left(\frac{\alpha}{4}\right)\right)+\chi_{2}\left(\left|\varphi_{l}\right|^{2}-\left|\varphi_{r}\right|^{2}\right) \sin \left(\frac{\alpha}{4}\right)\left(\frac{5}{2} \cos ^{2}\left(\frac{\alpha}{4}\right)-1\right) \\
+\frac{\sqrt{3}}{2} \cos \left(\frac{\alpha}{4}\right) G_{2}\left(\varphi^{*} \varphi_{l}+\varphi_{l}^{*} \varphi-\varphi^{*} \varphi_{r}+\varphi_{r}^{*} \varphi\right), \\
\quad=\quad k\left[\left(2 W_{d}-W_{r}-W_{l}\right)+\sqrt{3}\left(\Omega_{r}+\Omega_{l}\right)\right]+k_{c}\left[\frac{\sqrt{3}}{4} \sin \left(\frac{\alpha}{2}\right)\left(2 C+C_{r}+C_{l}\right)\right] \\
+\quad \chi_{1} \frac{1}{2}\left(2\left|\varphi_{d}\right|^{2}-\left|\varphi_{r}\right|^{2}-\left|\varphi_{l}\right|^{2}\right)+\chi_{2}\left(2|\varphi|^{2}+\left|\varphi_{r}\right|^{2}+\left|\varphi_{l}\right|^{2}\right)\left(\frac{\sqrt{3}}{4} \sin \left(\frac{\alpha}{2}\right)\right) \\
+\frac{1}{2} G_{2}\left(-\varphi^{*} \varphi_{r}-\varphi_{r}^{*} \varphi-\varphi^{*} \varphi_{l}-\varphi_{l}^{*} \varphi+2 \varphi^{*} \varphi_{d}+2 \varphi_{d}^{*} \varphi\right) \\
=\quad k\left[\sqrt{3} \sin \left(\frac{\alpha}{4}\right)(W r+W l)+\sin \left(\frac{\alpha}{4}\right)\left(\Omega_{r}+\Omega_{l}\right)\right]
\end{gathered}
$$




$$
\begin{aligned}
& +\quad k_{c}\left[\left(\frac{3}{2} \cos \left(\frac{\alpha}{4}\right)-\frac{5}{2} \cos ^{3}\left(\frac{\alpha}{4}\right)\right)\left(C_{r}+C_{l}\right)-\cos \left(\frac{\alpha}{4}\right) C_{d}+3 \cos ^{3}\left(\frac{\alpha}{4}\right) C\right] \\
& +\quad \chi_{1} \frac{\sqrt{3}}{2} \sin \left(\frac{\alpha}{4}\right)\left(2|\varphi|^{2}+\left|\varphi_{r}\right|^{2}+\left|\varphi_{l}\right|^{2}\right) \\
& +\quad \chi_{2}\left(\left(\frac{3}{2} \cos \left(\frac{\alpha}{4}\right)-\frac{5}{2} \cos ^{3}\left(\frac{\alpha}{4}\right)\right)\left(\left|\varphi_{r}\right|^{2}+\left|\varphi_{l}\right|^{2}\right)-\cos \left(\frac{\alpha}{4}\right)\left|\varphi_{d}\right|^{2}+3 \cos ^{3}\left(\frac{\alpha}{4}\right)|\varphi|^{2}\right) \\
& +G_{2} \frac{\sqrt{3}}{2} \sin \left(\frac{\alpha}{4}\right)\left(\varphi^{*} \varphi_{r}+\varphi_{r}^{*} \varphi+\varphi^{*} \varphi_{l}+\varphi_{l}^{*} \varphi\right) .
\end{aligned}
$$

Here we have used the notation $\varphi, \varphi_{r}, \varphi_{l}, \varphi_{d}$ for the functions on the lattice site $\{i, j, \rho\}$ and on its corresponding 3 neighbours.

\section{$3 \quad$ Numerical results}

In this section we describe various numerical stationary solutions of our equations. Note that our equations possess several parameters: $J, k, k_{c}, G_{2}, \chi_{i}$ and the lattice spacing $d$. For the numerical calculations we need dimensionless quantities and so we perform the following change of variables:

$$
\begin{aligned}
& u=l U ; \quad v=l V ; \quad s=l S ; \quad k=\frac{\kappa}{l^{2}} J ; \quad k_{c}=\frac{\kappa_{c}}{l^{2}} J \\
& \chi_{1}=\frac{X_{1}}{l^{2}} J ; \quad \chi_{2}=\frac{X_{2}}{l^{2}} J ; \quad G_{2}=\frac{G}{l^{2}} J ; \quad \mathcal{E}_{0}=\tilde{\mathcal{E}}_{0} J .
\end{aligned}
$$

First we choose to measure the energy in units of $J$ and so we put $J=1$. Then $\mathcal{E}_{0}$ is dimensionless. Next we choose the unit of length $l$ to be such that $\kappa=1$. From the expressions above we see that this corresponds to $l=\sqrt{J / k}$. For the carbon nanotube the parameters take the following values $J \approx 2.4 \mathrm{eV}[2,20,4], d=1.42 \times 10^{-10} \mathrm{~m}[2], k=36.5 \times 10^{4} \mathrm{dyn} / \mathrm{cm}$ [2]. Using these values, we get $l=(3.24-3.7) \times 10^{-11} \mathrm{~m}$. Thus, the length scale we use is smaller than the lattice length $d \approx 4 l$. For carbon nanotubes the dimensionless electron-phonon coupling constant $G=G_{2} l / J \sim 0.6-0.74$.

We have derived numerical solutions of our model for all values of $n$ between 3 and 20 for several ranges of other parameters. In all our calculations we have taken $k=1, k_{c}=0.2$. Then fixing the values of two of the three parameters $X_{1}, X_{2}$ and $G$ we have varied the value of the 3rd one between 0 and 5 , and $\chi_{1}$ and $\chi_{2}$ mostly between 0 and 1 . We have also set the lattice parameter $a=1$ and, in our discussions, we will refer to the main axis of the nanotube as $y$ while $x$ will run along the circumferences of the nanotube.

Of course, we have used periodic boundary conditions along the $x$ axis, while for the ends of the nanotube we have used free boundary conditions. This allows not only for a global translation of the nanotube but also for a dilation or contraction of the lattice along its axis. To exclude global translation of the lattice, we have pinned a single lattice site, at the bottom of the lattice, using a quadratic potential. Using such a potential, rather than fixing the position to a fixed value, is smoother from the numerical point of view.

\subsection{Numerical solutions}

We have found that, depending on the values of the parameters, the system of equations (19-22) possesses several types of solutions. Some of them, sometimes, coexist at the same values of the coupling constant. Some of these solutions are shown in Figs. 3-9 where we have plotted the hexagonal lattice unfolded onto a plane. The periodic boundary conditions imply that the left and right hand sides of the lattice are connected together (the lines joining these sites are not drawn in our figures, they are implicit). The position of each site is given by its equilibrium value translated by the deformations $u$ and $v$ at that lattice site. The $s$ field, on the other hand, 
is represented by an arrow, on the same scale, which points downwards if the $s$ field points towards the centre of the tube, i.e. if the tube is pinched, or upwards if the field points away from the tube, i.e. if the tube is dilated.

Most of the solutions we present here correspond to the $n=8$ case but the solutions for other values of $n$ look very much the same. Occasionally we will add some comments on this dependence. In the figures we only present a small section of the nanotube we have taken; for clarity of the picture we do show the full width of the tube, along its periodic direction, but we restrict ourselves to the section of the tube along its axis where the soliton is located. The solutions were actually computed on a grid ranging from -50 to +50 .

In our plots we have used the dimensionless units described above, which correspond to displacements 4 times larger than the real displacements of the nanotube, except for Figure 3.a where we had to amplify the dimensionless units by a factor of 10 and Figure 4.b where we have used the physical units because the displacements in this case were already sufficiently large. In the figures, each lattice site is represented by a circle, the inside of which, is painted in shades of black describing the value of the polaron probability density $|\varphi|^{2}$. A white inside of the circle corresponds to a very small density, while black is used for the largest density. The actual shade scale is given on the right hand side of each figure. Note that the axis of the nanotube runs vertically in our figures.
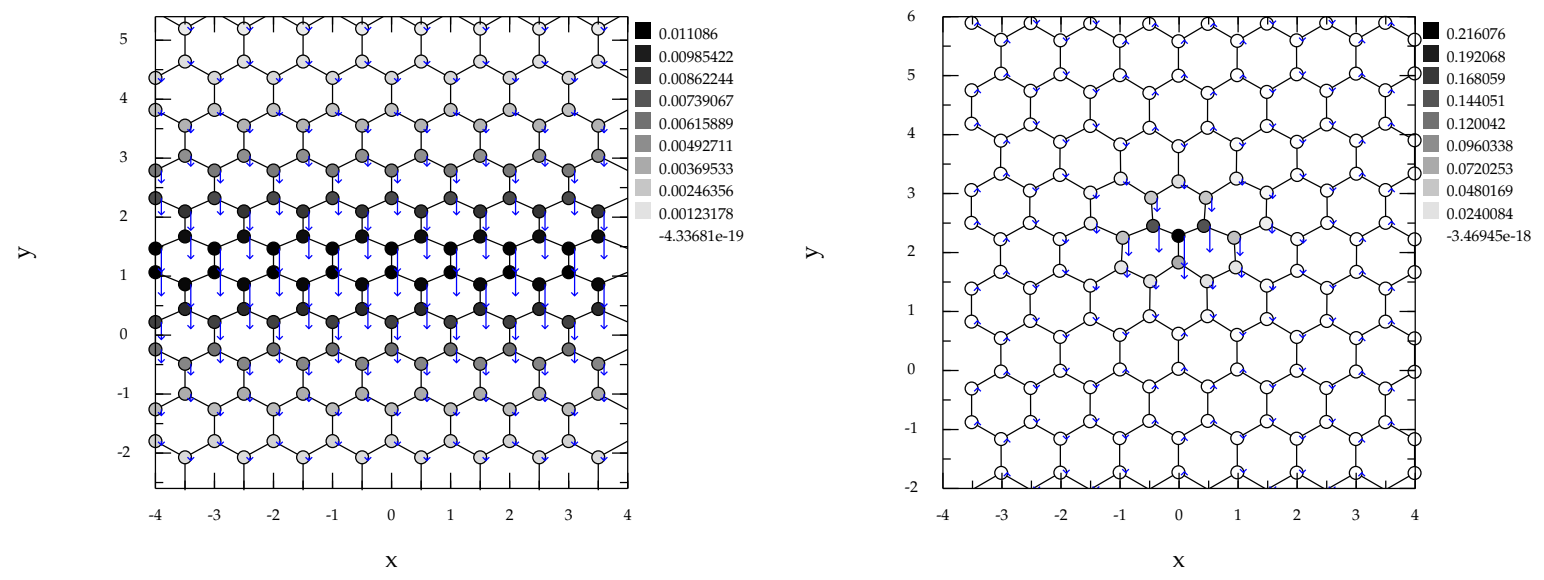

Figure 3: Solution for $n=8, X_{1}=0.6, X_{2}=0.2$ and a) $G=1$. b) $G=1.5$. The deformed lattice is plotted with small circles at the vertices representing the electron density (see the grey scale on the right; white corresponding to $|\varphi|^{2}=0$ ). The arrows at each vertex represent the $s$ field; a down/up arrow represents a displacement towards/away from the centre of the tube. The displacements are plotted in dimensionless units for $G=1.5$ and amplified by a factor of 10 for $G=1$.

When the electron-phonon coupling constants are small the solutions are invariably 1D bellshape solitons wrapped around the nanotube. This is seen in Fig. 3.a where the amplitudes of the displacements have been amplified 40 times compared to their physical values. In what follows, we name these solutions - type I solutions. We see that for type I solutions the polaron probability distribution looks like a 1D Davydov soliton along the axis of the nanotube which is spread uniformly around it. For our choice of parameters the nanotube is contracted at the position of the soliton and the tube itself is also pinched. Note that if we choose the 3 electronphonon coupling constants with the opposite signs, the displacements fields $u, v$ and $s$ will 
change signs and the nanotube will become extended and inflated.

Note also that the soliton drags the whole nanotube towards itself. In other words, the contraction of the lattice at the soliton position is not compensated by an appropriate dilation elsewhere.

When we increase the electron-phonon coupling constants above some critical value, the radially symmetrical solutions do not correspond to the minimum value of energy and the polaron localises on a few sites. We show some typical solutions in Figs. 3.b, 4 and 9. The solution at $X_{1}=0.6, X_{2}=0.2, G=1.5$ is shown in Fig. 3.b): the polaron probability density reaches a maximum on a single lattice site, but the polaron is spread over several lattice sites around the position of its maximum density. The lattice is slightly contracted but the lattice sites, where the polaron is localised, are slightly distorted into the nanotube. We name such solutions - 'the type II solutions'.
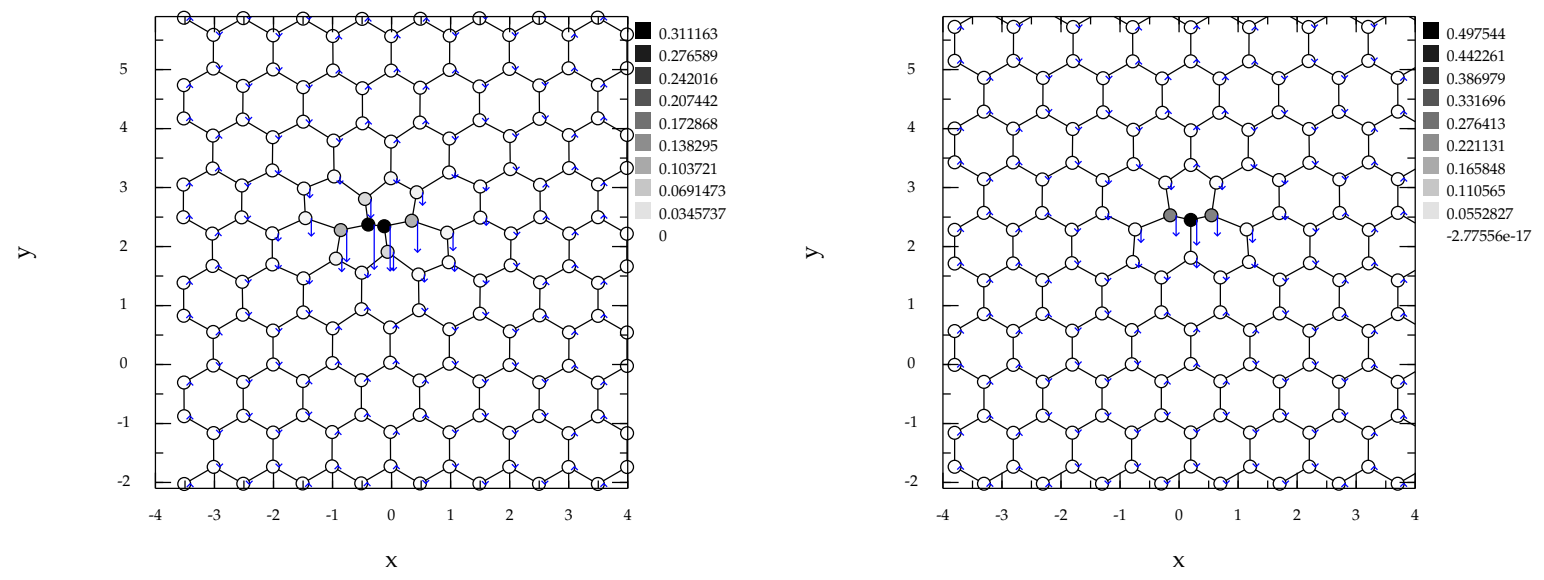

Figure 4: Solution for $n=8, X_{1}=0.6, X_{2}=0.2$ and a) $G=2$ b) $G=5$. The deformed lattice is plotted with small circles at the vertices representing the electron density (see the grey scale on the right; white corresponding to $|\varphi|^{2}=0$ ). The arrows at each vertex represent the $s$ field; a down/up arrow represents a displacement towards/away from the centre of the tube. The displacements are plotted in dimensionless units for $G=2$ and in physical units (dimensionless units divided by 4) for $G=5$.

In Fig. 4.a, we present a solution which is also localised on a few sites but for which the maximum of the polaron density is evenly distributed between 2 lattice sites. This solution corresponds to the same values of parameters as figure 3.b except that $G=2$. In this case, the lattice is also contracted, the polaron is more localised, and the lattice sites where the polaron is localised are also moved into the nanotube. Notice that the two sites at which the polaron density reaches its maximum have moved to be very close to each other. We name such solutions - 'the type III solutions'.

In Fig. 4.c, we present a solution corresponding to the same values of the parameters as figures 3.b and 4.a but for which $G=5$. This time the polaron is localised mainly on 3 lattice sites: it has a probability density of about 0.5 on one site and of 0.25 on either of the two neighbouring sites. The lattice deformation in this case is very large and so we had to scale it down to the physical scale. Once again, the lattice is contracted at the position of the polaron and the lattice sites where the polaron is located have all moved into the nanotube. We name these solutions - 'the type IV solutions'. 
The four classes of solutions we have presented so far, correspond to the same family of solutions obtained when one varies $G$ and takes $X_{1}=0.6$ and $X_{2}=0.2$. In Figs. 5-7 we present the maxima of the polaron probability densities $\left(D=\operatorname{Max}_{(i, j, \rho)}\left|\varphi_{i, j, \rho}\right|^{2}\right)$ and the eigenvalues $\Lambda$ of these solutions as a function of $G$. We present the data for the cases of $n=20,8$ and 3 . The solutions shown in figures 3 and 4 correspond to the 4 types of configurations presented in figures 6 . In particular we note that the transitions between the different types of solutions are always sharp and that sometimes there is an overlap: two different types of solutions coexist for the same values of the parameters.

We also note that the diameter of the nanotube does not affect very much the types of solutions that exist, except for the location of the transition between the different types of solutions. On the other hand, the binding energies of the solutions are larger for smaller values of $n$.
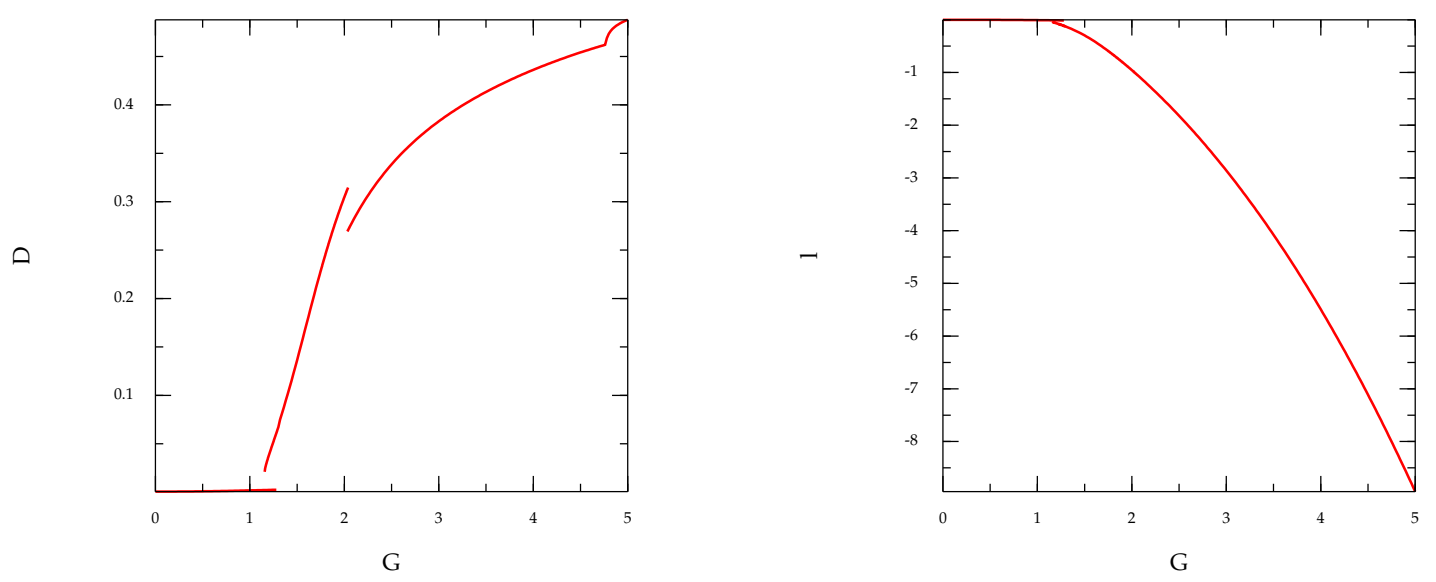

Figure 5: The maximum of probability, $D=\operatorname{Max}_{(i, j, \rho)}\left|\varphi_{i, j, \rho}\right|^{2}$ and the eigenvalue $\Lambda$ for $X_{1}=0.6$, $X_{2}=0.2$ as a function of $G$ for $n=20$.

In Fig. 8 we present the values of $G$ for which the various types of solutions exist as a function of $n$. We see that there is an overlap between solutions of type I and II and that this overlap is larger for larger values of $n$.

In Fig. 9.a, $X_{1}=2, X_{2}=0, G=0.6$ we present a solution which appears to be rather different from the previous ones. This time, the polaron is localised strongly on 4 sites, but with a density of about 0.55 at the central site. Note that the deformation of the lattice is nearly symmetric this time. A slight asymmetry is induced by the bending of the nanotube. We name these solutions - 'the type V solutions'.

Finally in Fig. 9.b we present a solution that is strongly localised on a single site. Note that this time the lattice is very strongly deformed. We name such solutions - 'the type VI solutions'.

\subsection{Solutions for $X_{1}=0.6, G=0.6$ and varying $X_{2}$}

In Fig. 10 we present the plots of the values of the maximum density and of the eigenvalues of the solutions for $X_{1}=0.6, G=0.6$ and $n=20$ as a function of $X_{2}$. We note that in this case all solutions fall into two classes seen before. The solutions with a small value of the maximum density are of type I while the highly peaked ones are of type VI. The solutions look very much the same for other values of $n$ except that the ranges of parameters for which the solutions exist do depend on $n$. 

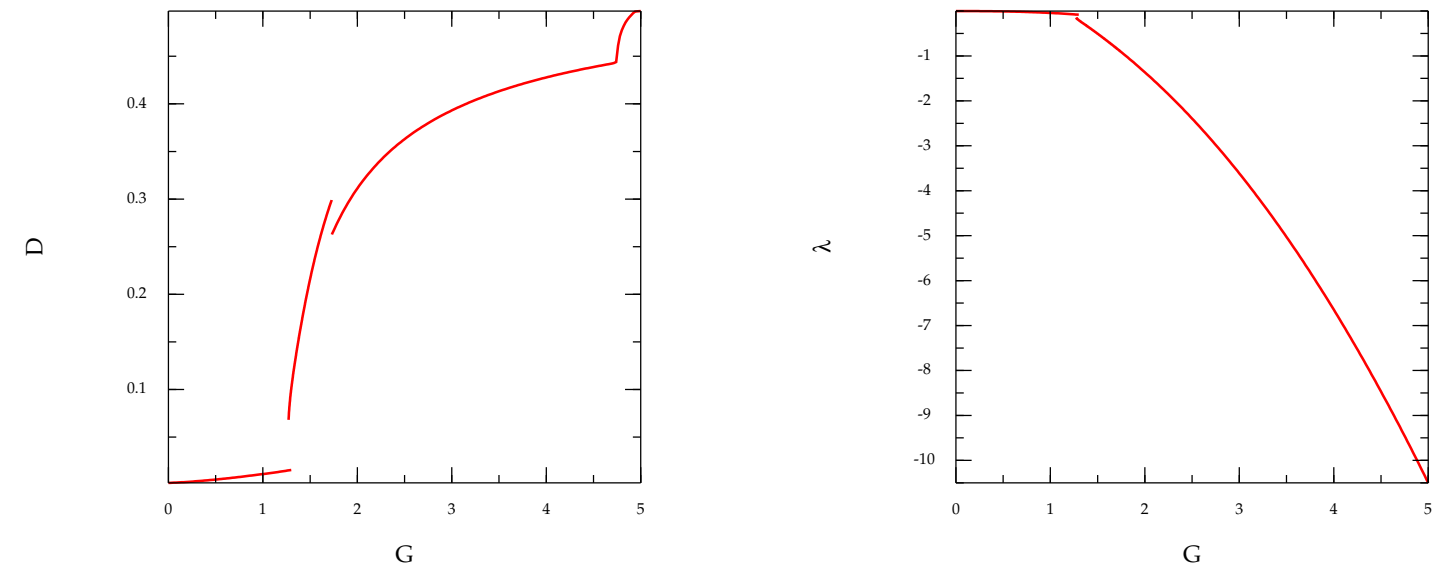

Figure 6: The maximum of probability, $D=\operatorname{Max}_{(i, j, \rho)}\left|\varphi_{i, j, \rho}\right|^{2}$ and the eigenvalue $\Lambda$ for $X_{1}=0.6$, $X_{2}=0.2$ as a function of $G$ for $n=8$.
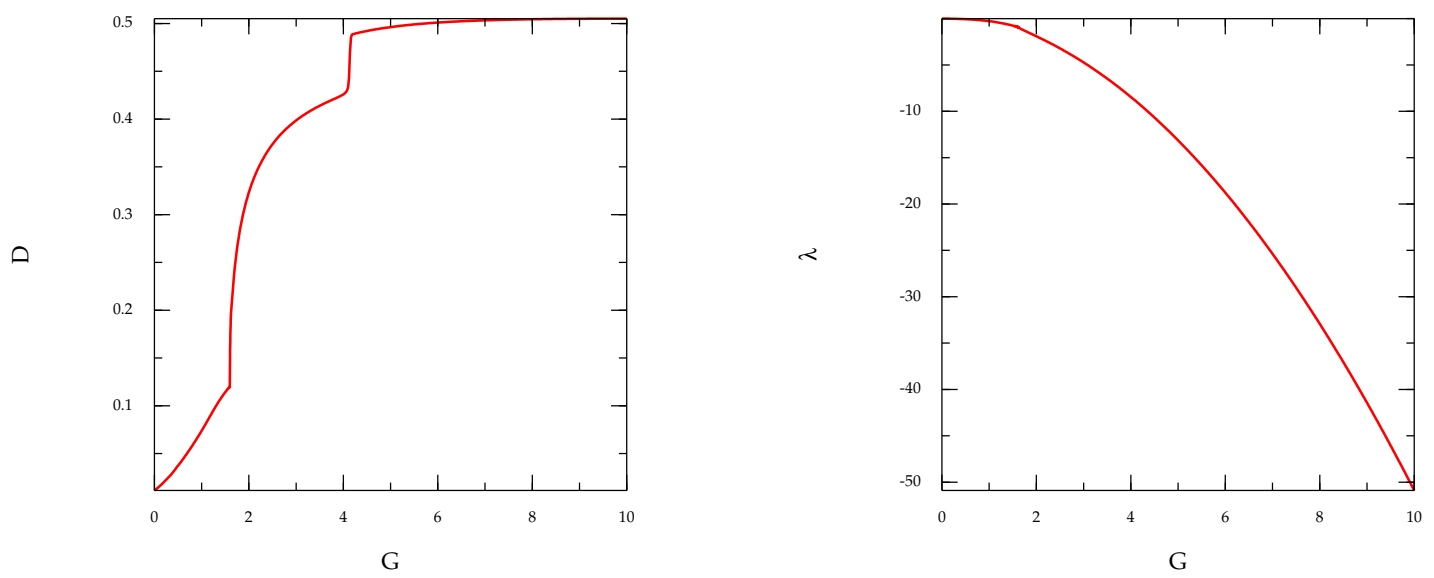

Figure 7: The maximum of probability, $D=\operatorname{Max}_{(i, j, \rho)}|\varphi|^{2}$ and the eigenvalue $\Lambda$ for $X_{1}=0.6$, $X_{2}=0.2$ as a function of $G$ for $n=3$. 


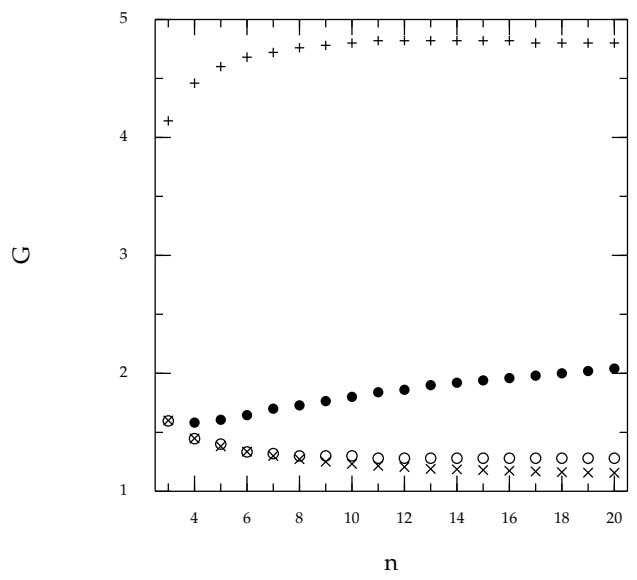

Figure 8: Domain of solutions as a function of $n$ for solutions with $X_{1}=0.6, X_{2}=0.2$. ०: upper value of $G$ for ring like solution (type I). × : lower value of $G$ for solutions of type II. • : Value of $G$ at the transition between solutions of type II and type III. + : Value of $G$ at the transition between solutions of type III and type IV.
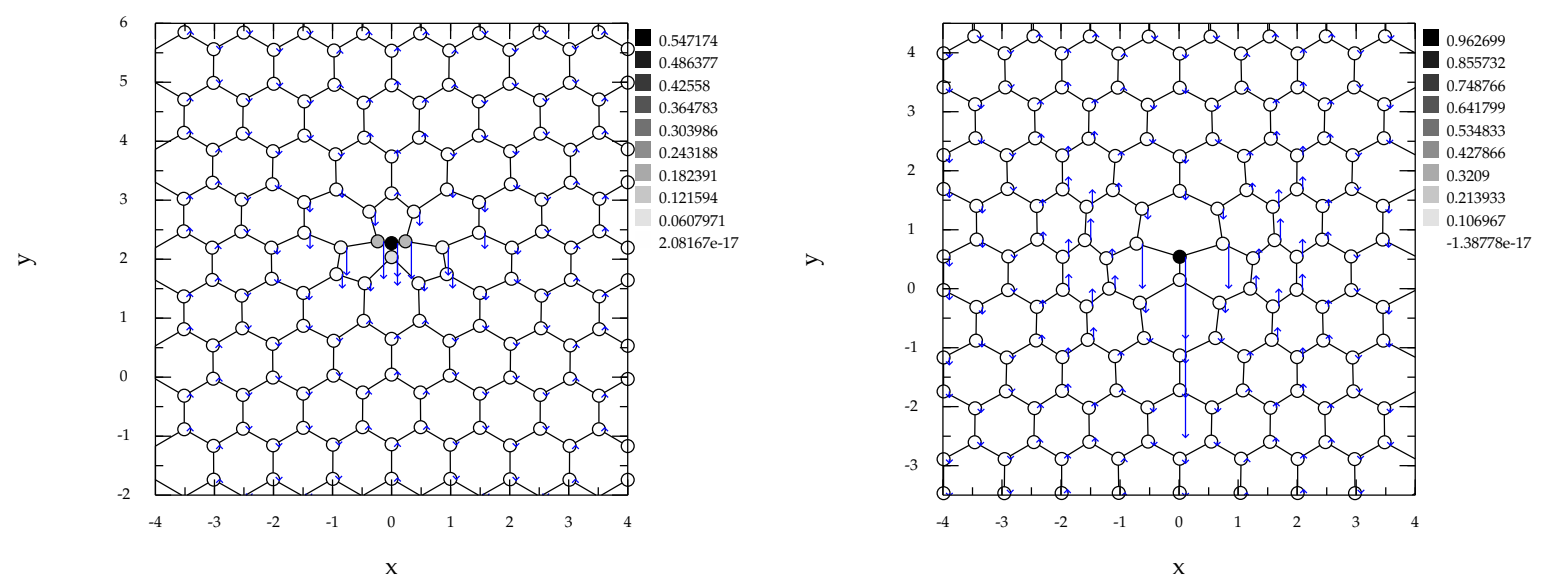

Figure 9: Solution for $n=8, G=0.6$ and a) $X 1=2, X_{2}=0$ b) $X 1=0.6, X_{2}=1.5$.

The deformed lattice is plotted with small circles at the vertices representing the electron density (see the grey scale on the right; white corresponding to $|\varphi|^{2}=0$ ). The arrows at each vertex represent the $s$ field; a down/up arrow represents a displacement towards/away from the centre of the tube. The displacements are plotted in dimensionless units. 

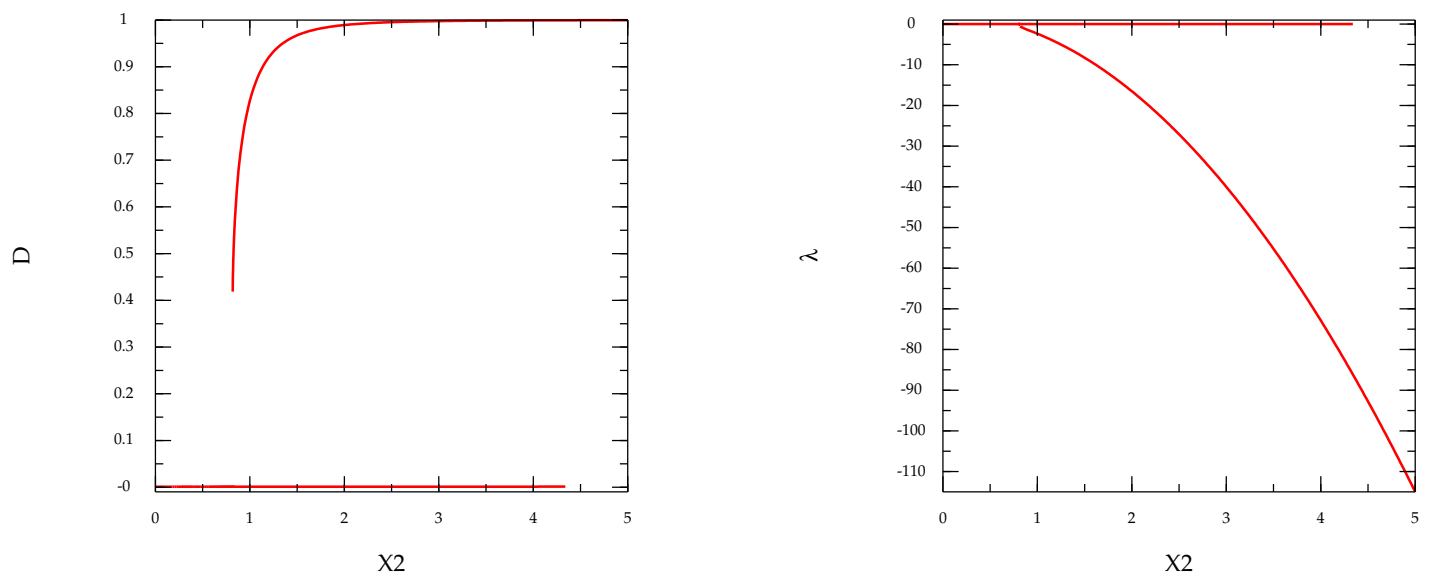

Figure 10: The maximum of probability, $D=\operatorname{Max}_{(i, j, \rho)}\left|\varphi_{i, j, \rho}\right|^{2}$ and the eigenvalue $\Lambda$ for $X_{1}=$ $0.6, G=0.6$ as a function of $X_{2}$ for $n=20$.

In Fig. 11 we present the regions of parameters for which the solutions of type I and type VI exist as a function of $n$ and $X_{2}$. Solutions of type I exist for all values of $X_{2}$ below the circles while those of type VI exist for all values of $X_{2}$ above the crosses.

We see that there is a large overlap of the regions in which the solutions of the type I and VI coexist and that the region of this overlap grows with $n$.

\subsection{Solutions for $X_{2}=0, G=0.6$ and various values of $X_{1}$}

In Fig. 12 we present the plots of the maximum density and of the eigenvalue of the solutions for $X_{2}=0, G=0.6$ and $n=20$ as a function of $X_{1}$. Again we note that there are two types of solutions in this case. The solutions with a small density maximum are of type I while the highly peaked ones are of type V. This time, however, there is very little overlap between these two classes of solutions.

The solutions look very much the same for other values of $n$, except that, again, the range of parameters where the concrete solutions exist does depend on $n$. For a given value of the parameters, the solutions are also more bound when $n$ is small.

From this numerical study and what we know about the physical values of the parameters in our model we can conclude that the type I solutions exist in all real nanotubes. If $X_{1}$ and $X_{2}$ are large, then strongly localised solutions of other types may exist as well.

\section{Conclusions}

In this paper we have determined the conditions for the existence of polaron (narrow soliton) states in hexagonal nanotube systems. They are given by the solutions of the equations minimising the total Hamiltonian of the system. We have also studied the properties of these solutions showing that in some cases the system possesses two or more different states very close in energy. Some of these states are localised on few lattice sites, some are more spread out. We have determined the boundaries between various states and have found that in some cases these boundaries are very sharp. The corresponding critical values of the coupling constants depend on the diameter of a nanotube. We have also shown that the solutions corresponding to the 


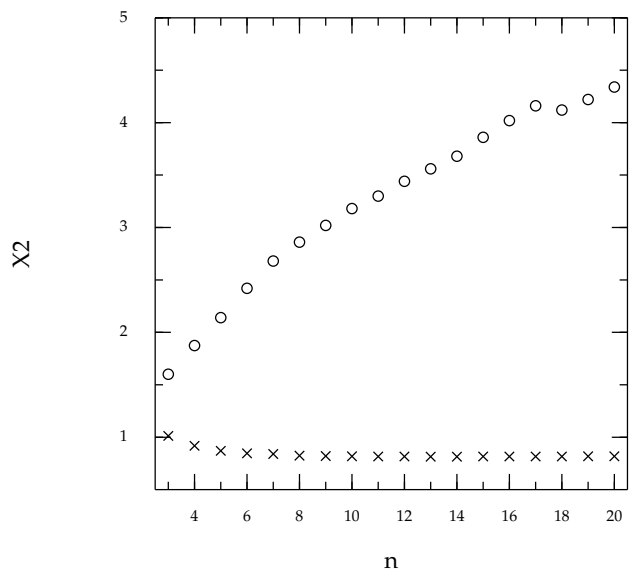

Figure 11: Domain of solutions as a function of $n$ for solutions with $X_{1}=0.6, G=0.6$. ०: upper value of $X_{2}$ for ring-like solutions (type I). × : lower value of $X_{2}$ for solutions of type VI.
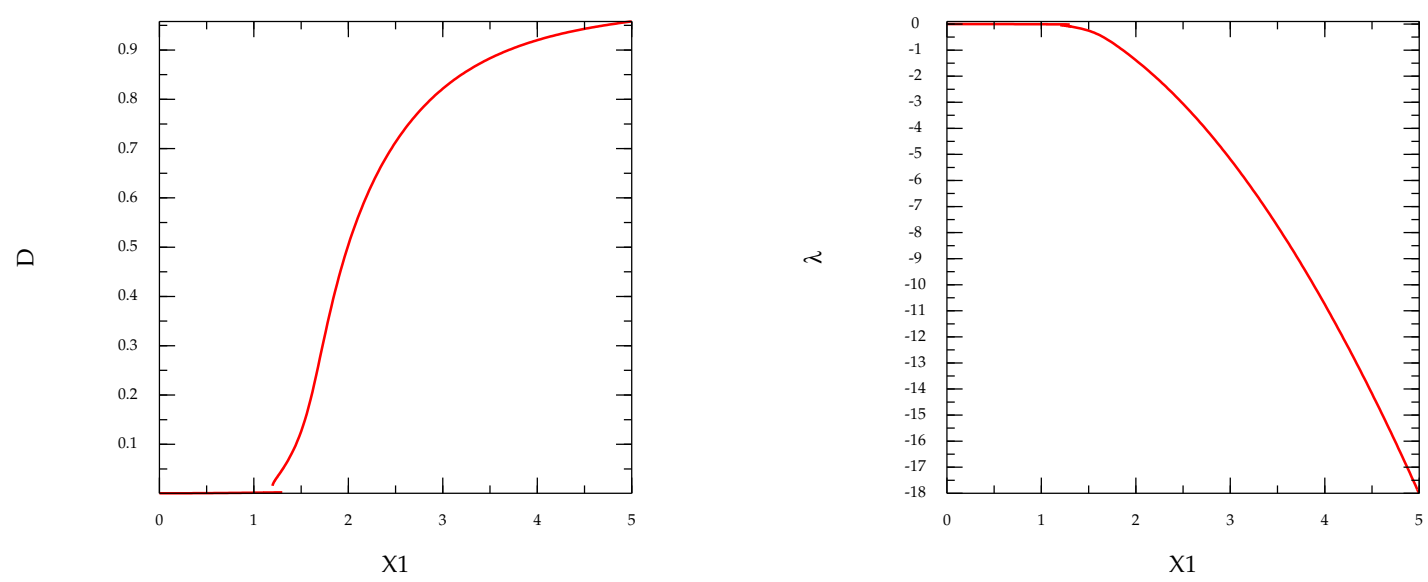

Figure 12: The maximum of probability, $D=\operatorname{Max}_{(i, j, \rho)}\left|\varphi_{i, j, \rho}\right|^{2}$ and the eigenvalue for $X_{2}=0$, $G=0.6$ as a function of $X_{1}$ for $n=20$. 
physical parameter values of the carbon nanotube are ring-like solutions wrapped around the nanotube with a profile resembling that of the nonlinear Schrödinger soliton, i.e. similar to a $1 \mathrm{D}$ soliton (comp. [15]).

Our numerical results do not apply straightforwardly to real carbon nanotubes with one electron per carbon atom when half of the electronic band states are occupied. Nevertheless, our results may be applicable to doped nanotubes at doping levels such that the concentration of charge carriers is sufficiently low so that the distances between self-trapped carriers exceed their localisation width. As has been shown in [23], in the case of the self-trapping, the Fermi statistics manifests itself by the appearance of the spatial repulsion of bipolarons. Two electrons with opposite spins can occupy the same place forming a bipolaron state. Two electrons with parallel spins or four electrons, in general, can create two self-trapped states. These states are characterised by a Fermi repulsion between them which decreases exponentially with the increases of the distance between them [23]. When the average distance between (bi)polarons is larger than the localisation region, the interaction between them can be neglected, and the single-(bi)polaron model, used in the present paper, becomes justified.

\section{Acknowledgement}

This work has been supported by a Royal Society travel grant.

\section{References}

[1] A.J. Heeger, S. Kivelson, J.R. Schrieffer, W.-P. Su, Rev. Mod. Phys. 60, 781 (1988).

[2] R. Saito, G. Dresselhaus and M.S. Dresselhaus, Physical Properties of Carbon Nanotubes (Imperial College Press, London, 1998).

[3] M.S. Dresselhaus, G. Dresselhaus and P.C. Ekland, Science of Fulerens and Carbon Nanotubes (Academic, New York, 1996).

[4] H. Dai, Surf. Sci. 500, 208 (2002).

[5] T. Hemraj-Benny, S. Bannerjee, et al., Phys. Chem. Chem. Phys. 7, 1103 (2005).

[6] H. Froehlich, Proc. Royal Soc. London Ser. A 215, 291 (1952).

[7] R.E. Peierls, Quantum Theory of Solids (Clarendon, Ozford, 1955).

[8] A.S. Davydov, Solitons in Molecular Systems (Dordrecht, Reidel, 1985).

[9] R. Saito, M. Fujita, G. Dresselhaus and M.S. Dresselhaus, Appl. Phys. Lett. 602204 (1992).

[10] J.W. Mintmire, B.I. Dunlap, and C.T. White, Phys. Rev. Lett. 68, 631 (1992).

[11] L. Duclaux, Carbon 40, 1751 (2002).

[12] W.P. Su, J.R. Schriefer, A.J. Heeger, Phys. Rev. Lett. 42, 1698 (1070); Phys. Rev. B 22, 2099 (1980).

[13] C.L. Kane, E.J. Mele, Phys. Rev. Lett., 78, 1932 (1997).

[14] C. Chamon, Phys. Rev. B 62, 2806 (2000).

[15] M. Verissimo-Alves, R.B. Capaz, B. Koiller, E. Artacho, and H. Chacham, Phys. Rev. Lett. 86, 3372 (2001). 
[16] Yu. Prylutskyy, A. Suprun, O. Ogloblya, in Book of abstracts of Intern. Conference on Theoretical Physics (Paris, UNESCO, July 22nd-27th, 2002) P.278; Yu.I. Prylutskyy, O.V. Ogloblya, P. Eklund, and P. Scharff, Synth. Met. (2001).

[17] L.M. Woods and G.D. Mahan, Phys. Rev. B 61, 10651 (2000).

[18] L.S. Brizhik, A.A. Eremko, B. Piette and W. Zakrzewski, Arxiv preprint cond-mat/0503366, (2005).

[19] R.A. Jishi, M.S. Dresselhaus, G. Dresselhaus, Phys. Rev. B 48, 11385 (1993).

[20] G.D. Mahan, Phys. Rev. B 68, 125409 (2003).

[21] L. Pietronero, S. Strässler, H.R. Zeller, M.J. Rice, Phys. Rev. B 22, 904 (1980).

[22] K. Kempa, Phys. Rev. B 66, 195406 (2002).

[23] L.S. Brizhik, A.A. Eremko, Physica D, 81, 295 (1995); Ukr. J. Phys., 44, 1022 (1999). 